\title{
SUBSONIC LAMB WAVES IN ANISOTROPIC PLATES
}

\author{
BY \\ SERGEY V. KUZNETSOV \\ Institute for Problems in Mechanics, Prosp. Vernadskogo, 101, Moscow, 117526 Russia
}

\begin{abstract}
A six-dimensional complex formalism for analysis of Lamb waves propagating with subsonic speed in anisotropic plates is formulated. Conditions for nonexistence of certain Lamb waves in anisotropic plates are obtained. An example of a transversely isotropic plate having "forbidden" speed at which no subsonic Lamb wave propagates is presented.
\end{abstract}

1. Introduction. Beginning from Lamb's pioneering work [1] in which the governing equations for harmonic waves propagating in isotropic plates with traction-free boundaries were first derived, in most of the subsequent theoretical works on Lamb waves in plates it was assumed that such a wave consists of several partial waves of the form

$$
\mathbf{u}_{k}(\mathbf{x})=\mathbf{m}_{k} e^{i r \gamma_{k} \mathbf{x} \cdot \nu} e^{i r(\mathbf{n} \cdot \mathbf{x}-c t)}
$$

where $\mathbf{u}_{k}$ is the displacement field of the $k$-th partial wave; $\mathbf{m}_{k}$ is the vectorial, in general, complex amplitude determined by the Christoffel equation (this equation will be introduced in Sec. 2); $\gamma_{k}$ is a root of the Christoffel equation; $r$ is the wave number; $\boldsymbol{\nu}$ is the unit normal to the middle plane of a plate; $\mathbf{n}$ is the unit vector determining the direction of propagation of the Lamb wave; $c$ is the phase speed; and $t$ is time. For existence of the resulting Lamb wave, all partial waves should have the same wave number and phase speed. Bearing in mind that the Christoffel equation has six roots, the representation for the Lamb wave takes the form

$$
\mathbf{u}(\mathbf{x})=\left(\sum_{k=1}^{6} C_{k} \mathbf{m}_{k} e^{i r \gamma_{k} \mathbf{x} \cdot \nu}\right) e^{i r(\mathbf{n} \cdot \mathbf{x}-c t)},
$$

where $C_{k}$ are arbitrary complex coefficients determined up to a multiplier by boundary conditions.

REMARK 1.1. a) Representation (1.1) for partial waves composing a Lamb wave is also used for analysis of Rayleigh waves propagating on the traction-free plane boundary of an elastic half space, see [2-8]; and for Stoneley interfacial waves propagating on the plane boundary between contacting dissimilar elastic half spaces, see [9-12].

Received December 4, 2000.

2000 Mathematics Subject Classification. Primary 74J15.

E-mail address: sv@kuznetsov.msk.ru

(C)2002 Brown University 
b) For Rayleigh waves, roots $\gamma_{k}$ in representation (1.1) should be complex with $\operatorname{Im}\left(\gamma_{k}\right)<0$. This ensures attenuation of the Rayleigh wave in the "lower" half-space $(\boldsymbol{\nu} \cdot \mathbf{x})<0$. If $\operatorname{Re}\left(\gamma_{k}\right)=0$ for all partial waves composing the Rayleigh wave, then such a wave is called a genuine Rayleigh wave; if $\operatorname{Re}\left(\gamma_{k}\right) \neq 0$ for some $k$, then it is sometimes called a generalized Rayleigh wave [8]. For Lamb waves, cases $\operatorname{Re}\left(\gamma_{k}\right)=0$ and $\operatorname{Re}\left(\gamma_{k}\right) \neq 0$ usually are not distinguished.

The following analysis indicates that for Lamb waves propagating with subsonic phase speed in anisotropic plates (subsonic phase speed does not exceed the minimal speed of all bulk waves propagating in the same direction), representation (1.2) needs correction: in some cases, depending on the anisotropy, the Lamb wave may consist of fewer components than is assumed in traditional approaches [13-16]. This phenomenon results in a statement asserting the possibility of existence of a "forbidden" subsonic speed, at which no Lamb wave can propagate. An example of the transversely isotropic plate having "forbidden" speed is constructed.

2. Basic notation. In the absence of body forces, the equation of motion for an anisotropic medium can be written in the form

$$
\mathbf{A}\left(\partial_{x}, \partial_{t}\right) \mathbf{u} \equiv \operatorname{div}_{x} \mathbf{C} \cdot \nabla_{x} \mathbf{u}-\rho \ddot{\mathbf{u}}=0,
$$

where $\rho$ is the material density and $\mathbf{C}$ is the fourth-order elasticity tensor assumed to be positive definite:

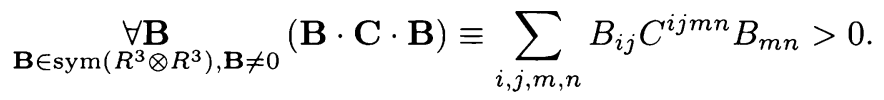

Substituting the partial wave (1.1) in Eq. (2.1) produces the Christoffel equation:

$$
\left(\left(\gamma_{k} \boldsymbol{\nu}+\mathbf{n}\right) \cdot \mathbf{C} \cdot\left(\mathbf{n}+\gamma_{k} \boldsymbol{\nu}\right)-\rho c^{2} \mathbf{I}\right) \cdot \mathbf{m}_{k}=0,
$$

where $\mathbf{I}$ is the unit diagonal matrix. Equation (2.3) admits an equivalent form:

$$
\operatorname{det}\left(\left(\gamma_{k} \boldsymbol{\nu}+\mathbf{n}\right) \cdot \mathbf{C} \cdot\left(\mathbf{n}+\gamma_{k} \boldsymbol{\nu}\right)-\rho c^{2} \mathbf{I}\right)=0 .
$$

The left-hand side of Eq. (2.4) can be regarded as a polynomial of degree six with respect to the parameter $\gamma_{k}$.

REMARK 2.1. a) Since polynomial coefficients in the left-hand side of Eq. (2.4) are real, corresponding roots $\gamma_{k}$ are either real or complex-conjugate.

b) It can be shown (see, for example, [5]) that Eq. (2.4) has no real roots if the phase speed is less than the so-called (lowest) limiting speed $c_{3}^{\lim }$. In its turn, $c_{3}^{\lim }$ does not exceed the lowest speed of all bulk waves propagating in the same direction. Hereinafter, the following condition will be imposed on the phase speed:

$$
0<c<c_{3}^{\lim }
$$

Condition (2.5) ensures absence of the real roots of Eq. (2.4). 
3. Six-dimensional formalism. Since it is not known in advance whether representation (1.1) for a partial wave is the only possible one, a more general representation for a harmonic wave with the plane wave front and nonhomogeneous amplitude will be considered:

$$
\mathbf{v}\left(x^{\prime \prime}\right) e^{i r(\mathbf{n} \cdot \mathbf{x}-c t)},
$$

where $\mathbf{v}\left(x^{\prime \prime}\right)$ is a non-constant complex-valued vector function; $x^{\prime \prime}=i r \nu \cdot \mathbf{x}$, and thus, $x^{\prime \prime}$ is a dimensionless (imaginary) coordinate in the direction determined by the vector $\boldsymbol{\nu}$. The exponential multiplier in (3.1) corresponds to movement of the plane wave front in the direction of propagation with the phase speed $c$. At this stage, no a priori restrictions on smoothness are imposed on the function $\mathbf{v}\left(x^{\prime \prime}\right)$.

Substituting representation (3.1) in Eq. (2.1) yields the following differential equation of the second order:

$$
\left((\boldsymbol{\nu} \cdot \mathbf{C} \cdot \boldsymbol{\nu}) \partial_{x^{\prime \prime}}^{2}+(\boldsymbol{\nu} \cdot \mathbf{C} \cdot \mathbf{n}+\mathbf{n} \cdot \mathbf{C} \cdot \boldsymbol{\nu}) \partial_{x^{\prime \prime}}+\left(\mathbf{n} \cdot \mathbf{C} \cdot \mathbf{n}-\rho c^{2} \mathbf{I}\right)\right) \mathbf{v}\left(x^{\prime \prime}\right)=0 .
$$

Direct analysis of Eq. (3.2) is difficult. The situation can be simplified by introducing an additional vector function

$$
\mathbf{w}\left(x^{\prime \prime}\right)=\partial_{x^{\prime \prime}} \mathbf{v}\left(x^{\prime \prime}\right) \text {. }
$$

Bearing in mind (3.3) and the positive-definite condition for the tensor C, Eq. (3.2) can be reduced to a matrix differential equation of the first order with respect to the six-dimensional vector function $(\mathbf{v}, \mathbf{w})$ :

$$
\begin{gathered}
\partial_{x^{\prime \prime}}\left(\begin{array}{l}
\mathbf{v} \\
\mathbf{w}
\end{array}\right)=\mathbf{R}_{6} \cdot\left(\begin{array}{l}
\mathbf{v} \\
\mathbf{w}
\end{array}\right), \\
\mathbf{R}_{6}=\left(\begin{array}{cc}
\mathbf{0} & \mathbf{I} \\
-\mathbf{M} & -\mathbf{N}
\end{array}\right) .
\end{gathered}
$$

In (3.4), real matrices $\mathbf{M}$ and $\mathbf{N}$ are as follows:

$$
\begin{aligned}
& \mathbf{M}=(\boldsymbol{\nu} \cdot \mathbf{C} \cdot \boldsymbol{\nu})^{-1} \cdot\left(\mathbf{n} \cdot \mathbf{C} \cdot \mathbf{n}-\rho c^{2} \mathbf{I}\right), \\
& \mathbf{N}=(\boldsymbol{\nu} \cdot \mathbf{C} \cdot \boldsymbol{\nu})^{-1} \cdot(\boldsymbol{\nu} \cdot \mathbf{C} \cdot \mathbf{n}+\mathbf{n} \cdot \mathbf{C} \cdot \boldsymbol{\nu}) .
\end{aligned}
$$

Taking into account the structure of the matrix $\mathbf{R}_{6}$, it is convenient to represent the corresponding six-dimensional eigenvectors of $\mathbf{R}_{6}$ in the form $\mathbf{m}_{6} \equiv\left(\mathbf{m} ; \mathbf{m}^{\prime}\right)$, where $\mathbf{m}, \mathbf{m}^{\prime} \in C^{3}$ (here $C^{k}$ denotes the $k$-dimensional complex vector space).

A surjective homomorphism $\mathfrak{I}: C^{6} \rightarrow C^{3}$ such that

$$
\mathfrak{I}\left(\mathbf{m}_{6}\right)=\mathbf{m}
$$

will be needed for the subsequent analysis.

REMARK 3.1. a) Due to (3.4), (3.5), the determinant of the matrix $\mathbf{R}_{6}$ can be represented in the form

$$
\operatorname{det} \mathbf{R}_{6}=\operatorname{det}\left((\boldsymbol{\nu} \cdot \mathbf{C} \cdot \boldsymbol{\nu})^{-1}\left(\mathbf{n} \cdot \mathbf{C} \cdot \mathbf{n}-\rho c^{2} \mathbf{I}\right)\right) .
$$

The right-hand side of (3.7) shows that matrix $\mathbf{R}_{6}$ is not degenerate at any phase speed with the exception when $\rho c^{2}=\lambda_{k}(\mathbf{n} \cdot \mathbf{C} \cdot \mathbf{n}), k=1,2,3$ (here $\lambda_{k}$ denotes an eigenvalue of the corresponding matrix); i.e., degeneracy occurs when the phase speed coincides with 
the speed of one of the bulk waves propagating in the same direction. It is clear that for the Lamb wave with the phase speed satisfying (2.5), matrix $\mathbf{R}_{6}$ is not degenerate.

b) Since matrix $\mathbf{R}_{6}$ is not symmetric, its left and right eigenvectors generally are different. In the subsequent analysis, the term "eigenvector" will refer to the right eigenvector of the matrix $\mathbf{R}_{6}$.

c) Matrix $\mathbf{R}_{6}$ resembles a matrix that is used for constructing the "fundamental elasticity tensor". Such a tensor and the corresponding matrix were introduced in [17] and were used later on for the analysis of Rayleigh and Stoneley waves propagating in anisotropic halfspaces; see $[4-8,11,12]$.

Proposition 3.1. a) The set of all roots of the Christoffel equation (2.4) coincides with the set of all eigenvalues of matrix $\mathbf{R}_{6}$.

b) The spectral space of the Christoffel equation (2.3) coincides with surjection (3.6) of the spectral space of matrix $\mathbf{R}_{6}$.

Proof. Let $\gamma_{k}$ be a root of the characteristic polynomial (2.4) and let $\mathbf{m}_{k}$ be the corresponding eigenvector of Eq. (2.3). Substituting functions $\mathbf{v}\left(x^{\prime \prime}\right)=\mathbf{m}_{k} e^{\gamma_{k} x^{\prime \prime}}$ and $\mathbf{w}\left(x^{\prime \prime}\right)=\gamma_{k} \mathbf{m}_{k} e^{\gamma_{k} x^{\prime \prime}}$ in Eq. (3.4) yields

$$
\gamma_{k}\left(\begin{array}{c}
\mathbf{m}_{k} \\
\gamma_{k} \mathbf{m}_{k}
\end{array}\right)=\mathbf{R}_{6} \cdot\left(\begin{array}{c}
\mathbf{m}_{k} \\
\gamma_{k} \mathbf{m}_{k}
\end{array}\right) .
$$

Thus, every root of Eq. (2.4) is an eigenvalue of matrix $\mathbf{R}_{6}$, and the corresponding eigenvector of Eq. (2.3) coincides with the vector $\mathfrak{I}\left(\mathbf{m}_{6}\right)$.

Further, let $\gamma_{k}$ be an eigenvalue, and $\left(\mathbf{m}_{k} ; \mathbf{m}_{k}^{\prime}\right), \mathbf{m}_{k}, \mathbf{m}_{k}^{\prime} \in C^{3}$, be the corresponding eigenvector of matrix $\mathbf{R}_{6}$ :

$$
\gamma_{k}\left(\begin{array}{c}
\mathbf{m}_{k} \\
\mathbf{m}_{k}^{\prime}
\end{array}\right)=\mathbf{R}_{6} \cdot\left(\begin{array}{c}
\mathbf{m}_{k} \\
\mathbf{m}_{k}^{\prime}
\end{array}\right) .
$$

Relation (3.9) along with (3.4), (3.5) yield

$$
\left(\gamma_{k}^{2}(\boldsymbol{\nu} \cdot \mathbf{C} \cdot \boldsymbol{\nu})+\gamma_{k}(\boldsymbol{\nu} \cdot \mathbf{C} \cdot \mathbf{n}+\mathbf{n} \cdot \mathbf{C} \cdot \boldsymbol{\nu})+\left(\mathbf{n} \cdot \mathbf{C} \cdot \mathbf{n}-\rho c^{2} \mathbf{I}\right)\right) \cdot \mathbf{m}_{k}=0
$$

But (3.10) coincides with (2.3).

Remark 2.1 ensures

COROllary. Under condition (2.5), all eigenvalues of matrix $\mathbf{R}_{6}$ are complex and form the spectrum of $\mathbf{R}_{6}$ by complex-conjugate pairs.

Proposition 3.2. Under condition (2.5), matrix $\mathbf{R}_{6}$ is not a normal matrix, except maybe one value of the phase speed $c$.

Proof. Definition of the normal (real) matrix gives

$$
\mathbf{R}_{6}^{t} \cdot \mathbf{R}_{6}=\mathbf{R}_{6} \cdot \mathbf{R}_{6}^{t}
$$


In view of (3.4), relation (3.11) implies

$$
\mathbf{M}^{t} \cdot \mathbf{M}=\mathbf{I}
$$

Taking into account (3.5), relation (3.12) requires

$$
\mathbf{n} \cdot \mathbf{C} \cdot \mathbf{n}-\boldsymbol{\nu} \cdot \mathbf{C} \cdot \boldsymbol{\nu}=\rho c^{2} \mathbf{I}
$$

This completes the proof, since the left-hand side of (3.13) is independent of $c$.

Corollary. Under condition (2.5), the eigenvectors of matrix $\mathbf{R}_{6}$ do not form an orthogonal basis in $C^{6}$, except maybe one value of the phase speed $c$ at which relation (3.13) holds.

4. Representations for Lamb waves. The structure of the general solution of system (3.4) is determined by the Jordan normal form of matrix $\mathbf{R}_{6}$ [18, Chap. IV, §5]. Due to Proposition 3.1 and the corresponding corollary, for the phase speed that satisfies (2.5), only three types of the Jordan normal forms of matrix $\mathbf{R}_{6}$ can occur:

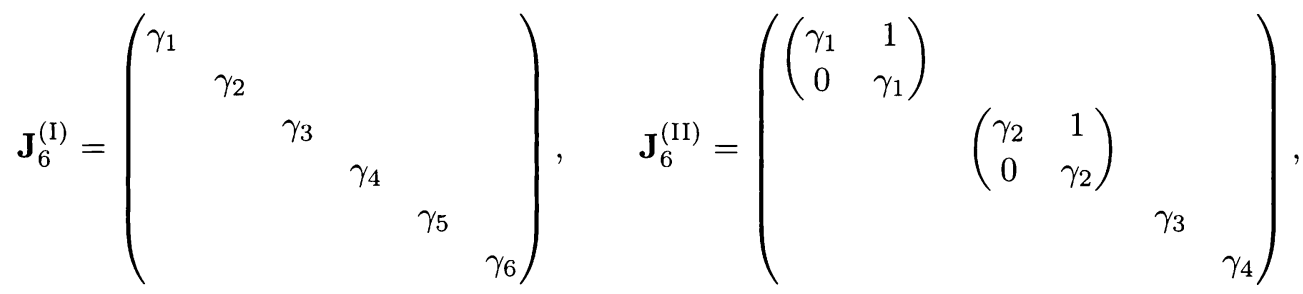

$$
\begin{aligned}
& \mathbf{J}_{6}^{(\mathrm{III})}=\left(\begin{array}{ccc}
\left(\begin{array}{ccc}
\gamma_{1} & 1 & 0 \\
0 & \gamma_{1} & 1 \\
0 & 0 & \gamma_{1}
\end{array}\right) & \left(\begin{array}{ccc}
\gamma_{2} & 1 & 0 \\
0 & \gamma_{2} & 1 \\
0 & 0 & \gamma_{2}
\end{array}\right)
\end{array}\right) .
\end{aligned}
$$

In expressions (4.1), eigenvalues $\gamma_{k}$ are renumbered in such a way that they satisfy the following condition:

$$
\gamma_{2 k-1}=\overline{\gamma_{2 k}}, \quad k=1,2, \ldots
$$

Moreover, since matrix $\mathbf{R}_{6}$ is real, a condition analogous to (4.2) is satisfied by eigenvectors of $\mathbf{R}_{6}$ :

$$
\left(\mathbf{m}_{2 k-1} ; \mathbf{m}_{2 k-1}^{\prime}\right)=\left(\overline{\mathbf{m}_{2 k}} ; \overline{\mathbf{m}_{2 k}^{\prime}}\right)
$$


Transition from the system of the first order (3.4) to the initial system (3.2) allows us to represent the general solution (the fundamental matrix) in the form:

$$
\begin{aligned}
\mathbf{v}^{(\mathrm{I})}\left(x^{\prime \prime}\right)= & \sum_{k=1}^{6} C_{k} \mathbf{m}_{k} e^{\gamma_{k} x^{\prime \prime}} \\
\mathbf{v}^{(\mathrm{II})}\left(x^{\prime \prime}\right)= & \sum_{k=1}^{2}\left(\left(C_{2 k-1}+C_{2 k} x^{\prime \prime}\right) \mathbf{m}_{k}+C_{2 k} \mathbf{m}_{k}^{1}\right) e^{\gamma_{k} x^{\prime \prime}}+\sum_{k=3}^{4} C_{k+2} \mathbf{m}_{k} e^{\gamma_{k} x^{\prime \prime}}, \\
\mathbf{v}^{(\mathrm{III})}\left(x^{\prime \prime}\right)= & \sum_{k=1}^{2}\left(C_{3 k-2}+C_{3 k-1} x^{\prime \prime}+\frac{1}{2} C_{3 k} x^{\prime \prime 2}\right) \mathbf{m}_{k} e^{\gamma_{k} x^{\prime \prime}} \\
& +\sum_{k=1}^{2}\left(\left(C_{3 k-1}+C_{3 k} x^{\prime \prime}\right) \mathbf{m}_{k}^{1}+C_{3 k} \mathbf{m}_{k}^{2}\right) e^{\gamma_{k} x^{\prime \prime}}
\end{aligned}
$$

where $C_{k}$ are unknown complex coefficients, and $\mathbf{m}_{k}^{1}, \mathbf{m}_{k}^{2} \in C^{3}$ are the generalized eigenvectors associated with the eigenvector $\mathbf{m}_{k}$.

5. Disperse equations. Traction-free boundary conditions are formulated on the plate surfaces

$$
\left.\mathbf{t}_{\boldsymbol{\nu}}\right|_{\mathbf{x} \cdot \boldsymbol{\nu}= \pm h} \equiv \pm\left.\boldsymbol{\nu} \cdot \mathbf{C} \cdot \nabla_{\mathbf{x}} \mathbf{u}\right|_{\mathbf{x} \cdot \boldsymbol{\nu}= \pm h}=0 .
$$

In (5.1), $2 h$ is the plate thickness.

Substituting the displacement field (4.4) in the boundary conditions (5.1) and making the transition to the dimensionless coordinate $x^{\prime \prime}=\operatorname{ir}(\boldsymbol{\nu} \cdot \mathbf{x})$ gives

$$
\left.\sum_{k=1}^{6} C_{k} \mathbf{t}_{k} e^{\gamma_{k} x^{\prime \prime}}\right|_{x^{\prime \prime}= \pm \xi}=0
$$

where $\xi=i r h$ and $\mathbf{t}_{k}$ is (up to the exponential multiplier) the surface traction corresponding to the coefficient $C_{k}$.

Solution of the boundary-value problem (5.2) can be treated as a nontrivial solution of the linear system (5.2) with respect to unknown coefficients $C_{k}, k=1, \ldots, 6$. The latter is equivalent to vanishing all the determinants of the sixth order associated with the $6 \times 6$ matrix:

$$
\delta \equiv \operatorname{det}\left(\begin{array}{ccc}
\mathbf{t}_{1}(\xi) e^{+\gamma_{1} \xi} & \cdots & \mathbf{t}_{6}(\xi) e^{+\gamma_{6} \xi} \\
\mathbf{t}_{1}(-\xi) e^{-\gamma_{1} \xi} & \cdots & \mathbf{t}_{6}(-\xi) e^{-\gamma_{6} \xi}
\end{array}\right)=0 .
$$

Equations (5.3) are the disperse equations that we are looking for. In the case of arbitrary elastic anisotropy, solvability of Eqs. (5.3) has not been studied; however, the following propositions hold.

Proposition 5.1. The condition

$$
\gamma_{k}=-\frac{\boldsymbol{\nu} \otimes \overline{\mathbf{m}}_{k} \cdot \mathbf{C} \cdot \mathbf{m}_{k} \otimes \mathbf{n}}{\boldsymbol{\nu} \otimes \overline{\mathbf{m}}_{k} \cdot \mathbf{C} \cdot \mathbf{m}_{k} \otimes \boldsymbol{\nu}}
$$

is necessary for the Lamb wave to be composed of a single partial wave (1.1). 
Proof. If at some $k$, the corresponding partial wave satisfies the boundary conditions, then Eqs. (5.2) yield

$$
\left(\gamma_{k} \boldsymbol{\nu} \cdot \mathbf{C} \cdot \boldsymbol{\nu}+\boldsymbol{\nu} \cdot \mathbf{C} \cdot \mathbf{n}\right) \cdot \mathbf{m}_{k}=0 .
$$

Multiplication of both sides of Eq. (5.5) by $\overline{\mathbf{m}}_{k}$ gives

$$
\gamma_{k} \boldsymbol{\nu} \otimes \overline{\mathbf{m}}_{k} \cdot \mathbf{C} \cdot \mathbf{m}_{k} \otimes \boldsymbol{\nu}+\boldsymbol{\nu} \otimes \overline{\mathbf{m}}_{k} \cdot \mathbf{C} \cdot \mathbf{m}_{k} \otimes \mathbf{n}=0
$$

Then, in view of the positive definiteness of the elasticity tensor, it remains to note that Eq. (5.6) is equivalent to (5.4).

Proposition 5.2. Condition (5.4) is necessary for the Lamb wave to be composed of two partial waves (1.1) corresponding to the complex-conjugate eigenvalues.

Proof. Assume that (5.4) does not hold, but a nontrivial solution of Eqs. (5.2) exists at some eigenvalues $\gamma_{k}$ and $\bar{\gamma}_{k}$. In this case, Eqs. (5.3) reduce to two equations. The first one can be represented in the form

$$
\mathbf{t}_{k} \times \overline{\mathbf{t}_{k}}=0,
$$

where $\mathbf{t}_{k}=\left[\gamma_{k} \boldsymbol{\nu} \cdot \mathbf{C} \cdot \boldsymbol{\nu}+\boldsymbol{\nu} \cdot \mathbf{C} \cdot \mathbf{n}\right] \cdot \mathbf{m}_{k}$. Equation (5.7) means colinearity of vectors $\mathbf{t}_{k}$ and $\overline{\mathrm{t}_{k}}$ :

$$
\mathbf{t}_{k}=\alpha \mathbf{e}, \quad \overline{\mathbf{t}_{k}}=\bar{\alpha} \mathbf{e},
$$

where $\alpha$ is a complex constant ( $\alpha \neq 0$, since (5.4) does not hold by the assumption) and $\mathbf{e} \in R^{3}$. Taking account of (5.8), the second equation flowing out from (5.3) takes the form

$$
\operatorname{det}\left(\begin{array}{ll}
\alpha e^{+\gamma_{k} \xi} & \bar{\alpha} e^{+\bar{\gamma}_{k} \xi} \\
\alpha e^{-\gamma_{k} \xi} & \bar{\alpha} e^{-\bar{\gamma}_{k} \xi}
\end{array}\right)=0 .
$$

Direct verification shows that for $\operatorname{Im}\left(\gamma_{k}\right) \neq 0$ and $\alpha \neq 0$, the left-hand side of (5.9) cannot vanish.

REMARK 5.1. a) Condition (5.4) is not sufficient for the Lamb wave to be composed of a single partial wave, or two partial waves corresponding to the complex-conjugate eigenvalues. This is because of the possibility for vectors $\overline{\mathbf{m}}_{k}$ and $\mathbf{t}_{k} \equiv\left[\gamma_{k} \boldsymbol{\nu} \cdot \mathbf{C} \cdot \boldsymbol{\nu}+\boldsymbol{\nu}\right.$ $\mathbf{C} \cdot \mathbf{n}] \cdot \mathbf{m}_{k} \neq 0$ to be mutually orthogonal.

b) Proposition 5.1 remains valid for Rayleigh waves. In this case, conditions (5.1), (5.2) are formulated on the free surface at $x^{\prime \prime}=0$. In an indirect proof, based on Stroh's formalism (see [4], [5]), it was shown that a subsonic Rayleigh wave cannot be composed of a single partial wave. For a supersonic Rayleigh wave propagating on a half-space with elastic symmetry, a condition analogous to (5.4) was derived in [19].

6. Lamb waves in transversely isotropic plates. Let the unit vectors $\mathbf{e}_{k}, k=$ $1,2,3$, form an orthogonal basis in $R^{3}$, where the vector $\mathbf{e}_{1}$ coincides with the normal vector $\boldsymbol{\nu}$ to the median surface of a plate, while vectors $\mathbf{e}_{2}$ and $\mathbf{e}_{3}$ lie on the basal plane 
of a transversely isotropic medium. The corresponding elasticity tensor has the following components:

$$
\begin{array}{cccccc}
c_{11} & c_{12} & c_{12} & 0 & 0 & 0 \\
& c_{22} & c_{23} & 0 & 0 & 0 \\
& & c_{22} & 0 & 0 & 0 \\
& & & c_{44} & 0 & 0 \\
& & & & c_{55} & 0 \\
& & & & & c_{55}
\end{array}
$$

where $c_{44}=\frac{1}{2}\left(c_{22}-c_{23}\right)$. The condition of positive definiteness for the regarded elasticity tensor yields

$$
c_{11}>0 ; \quad c_{22}>0 ; \quad c_{55}>0 ; \quad c_{22}>\left|c_{23}\right| ; \quad c_{11} c_{22}>c_{12}^{2} ; \quad c_{11}>\frac{2 c_{12}^{2}}{c_{22}+c_{23}} .
$$

Substituting the elasticity tensor (6.1) in (3.5) gives

$$
\begin{aligned}
& \mathbf{M}=\left(\frac{c_{55}-\rho c^{2}}{c_{11}} \boldsymbol{\nu} \otimes \boldsymbol{\nu}+\frac{c_{22}-\rho c^{2}}{c_{55}} \mathbf{n} \otimes \mathbf{n}+\frac{c_{22}-c_{23}-\rho c^{2}}{2 c_{55}} \mathbf{w} \otimes \mathbf{w}\right), \\
& \mathbf{N}=\left(\frac{c_{12}+c_{55}}{c_{11}} \boldsymbol{\nu} \otimes \mathbf{n}+\frac{c_{12}+c_{55}}{c_{55}} \mathbf{n} \otimes \boldsymbol{\nu}\right),
\end{aligned}
$$

where $\mathbf{w}=\mathbf{n} \times \boldsymbol{\nu}$.

Taking account of (6.3), the following proposition flows out directly from the analysis of the spectral properties of matrix $\mathbf{R}_{6}$.

Proposition 6.1. a) The relation between the elastic constants, density, and phase speed

$$
\begin{aligned}
\rho c^{2}=2 & \frac{\left|c_{12}+c_{55}\right|}{\left(c_{11}-c_{55}\right)^{2}} \sqrt{c_{11} c_{55}\left(c_{11} c_{55}+c_{22} c_{55}+2 c_{12} c_{55}-c_{11} c_{22}+c_{12}^{2}\right)} \\
& -\frac{2 c_{12} c_{55}^{2}+2 c_{11} c_{12} c_{55}-c_{11}^{2} c_{22}+c_{11} c_{22} c_{55}+c_{11} c_{12}^{2}+c_{12}^{2} c_{55}+2 c_{11} c_{55}^{2}}{\left(c_{11}-c_{55}\right)^{2}}
\end{aligned}
$$

is necessary and sufficient for the rise of the Jordan normal form $\mathbf{J}_{6}^{(\mathrm{II})}$.

b) Different roots $\gamma_{k}$ of the Christoffel equation (2.4) corresponding to (6.4) are

$$
\begin{array}{ll}
\gamma_{1}=-i\left(\frac{c_{11} c_{22}-\left(c_{11}+c_{55}\right) \rho c^{2}-2 c_{12} c_{55}-c_{12}^{2}}{2 c_{11} c_{55}}\right)^{1 / 2}, & \gamma_{2}=-\gamma_{1}, \\
\gamma_{3}=-i\left(\frac{c_{22}-c_{23}-2 \rho c^{2}}{2 c_{55}}\right)^{1 / 2}, & \gamma_{4}=-\gamma_{3},
\end{array}
$$

where $\gamma_{1}$ and $\gamma_{2}$ correspond to the Jordan blocks. 
c) Corresponding amplitudes have the form

$$
\begin{array}{lll}
\mathbf{m}_{1}=p \boldsymbol{\nu}\left(\frac{c_{22}-\rho c^{2}}{c_{11}}\right)^{1 / 4}+i p \mathbf{n}\left(1-\frac{\rho c^{2}}{c_{55}}\right)^{1 / 4}, & \mathbf{m}_{2}=\overline{\mathbf{m}}_{1}, \\
\mathbf{m}_{1}^{1}=i p \boldsymbol{\nu}\left(1-\frac{\rho c^{2}}{c_{55}}\right)^{1 / 4}+p \mathbf{n}\left(\frac{c_{22}-\rho c^{2}}{c_{11}}\right)^{1 / 4}, & \mathbf{m}_{2}^{1}=\overline{\mathbf{m}}_{1}^{1}, \\
\mathbf{m}_{3}=\mathbf{m}_{4}=\mathbf{w}, &
\end{array}
$$

where $p$ is the normalization factor:

$$
p=\left(\left(\frac{c_{22}-\rho c^{2}}{c_{11}}\right)^{1 / 2}+\left(1-\frac{\rho c^{2}}{c_{55}}\right)^{1 / 2}\right)^{-1 / 2} .
$$

REMARK 6.1. a) The natural requirement for the right-hand side of (6.4) to be real and positive leads to the following restrictions:

$$
\begin{array}{cl}
c_{11} \neq c_{55} ; \quad & c_{12}^{2}+c_{11} c_{55}+c_{22} c_{55}+2 c_{12} c_{55}-c_{11} c_{22} \geq 0 \\
& c_{12}^{2}+4 c_{12} c_{55}+4 c_{55}^{2}-c_{11} c_{22}>0 .
\end{array}
$$

In (6.4)-(6.8) and hereinafter, the case $c_{11}=c_{55}$ is not considered. Of course, conditions (6.8) should be completed with (6.2) and (2.5). Direct verification shows that, all together, conditions (6.8), (6.2) and (2.5) define the nonempty region $\Omega \subset R^{5}$ of admissible values of elastic parameters.

Finally, the main result of this section can be proved.

TheOREM 6.1. No Lamb wave propagates in the transversely isotropic plate if the phase speed satisfies condition (6.4).

Proof. Substituting eigenvalues (6.5) and corresponding amplitudes (6.6) in (5.3) gives two independent conditions:

$$
\operatorname{det}_{+}\left(\begin{array}{lcc}
\left(a_{1} \boldsymbol{\nu}+b_{1} \mathbf{n}\right) e^{+\gamma_{1} \xi}\left(\bar{a}_{1} \boldsymbol{\nu}+\bar{b}_{1} \mathbf{n}\right) e^{+\bar{\gamma}_{1} \xi} & \left(a_{2}(\xi) \boldsymbol{\nu}+b_{2}(\xi) \mathbf{n}\right) e^{+\gamma_{1} \xi} & \left(\overline{a_{2}(\xi)} \boldsymbol{\nu}+\overline{b_{2}(\xi)} \mathbf{n}\right) e^{+\bar{\gamma}_{1} \xi} \\
\left(a_{1} \boldsymbol{\nu}+b_{1} \mathbf{n}\right) e^{-\gamma_{1} \xi}\left(\overline{a_{1}} \boldsymbol{\nu}+\bar{b}_{1} \mathbf{n}\right) e^{-\bar{\gamma}_{1} \xi} & \left(a_{2}(-\xi) \boldsymbol{\nu}+b_{2}(-\xi) \mathbf{n}\right) e^{-\gamma_{1} \xi}\left(\overline{a_{2}(-\xi)} \boldsymbol{\nu}+\overline{b_{2}(-\xi)} \mathbf{n}\right) e^{-\bar{\gamma}_{1} \xi}
\end{array}\right)=0
$$

and

$$
\operatorname{det}_{2}\left(\begin{array}{ll}
(d \mathbf{w}) e^{+\gamma_{3} \xi} & (\bar{d} \mathbf{w}) e^{+\bar{\gamma}_{3} \xi} \\
(d \mathbf{w}) e^{-\gamma_{3} \xi} & (\bar{d} \mathbf{w}) e^{-\bar{\gamma}_{3} \xi}
\end{array}\right)=0
$$

where $a_{1}, b_{1}, a_{2}, b_{2}, d$ are scalar (nonzero) complex coefficients determined by (6.5), (6.6), and (5.3). Finally, direct computation of the determinants in the left-hand sides of $(6.9)$ and $(6.10)$ reveals that both of these determinants do not vanish at nonzero $\xi$. This completes the proof.

REMARK 6.2. a) A more detailed analysis shows that "forbidden" speed for a transversely isotropic plate does not depend upon the wave frequency and thickness of a plate. As follows from (6.4), such a speed is determined only by the physical properties of a material.

b) It can be highly important for practical applications (for example, design of the delay lines and filters in electronics) that some transversely isotropic materials that only 
slightly differ from isotropic ones can have "forbidden" speed for Lamb waves. For instance, taking in (6.2), (6.8),

$$
c_{11}=c_{22}=1 ; \quad c_{55}=\frac{1}{2} ; \quad c_{23}=0,
$$

we arrive at the following restriction imposed on $c_{12}$ :

$$
\frac{\sqrt{2}}{2}>c_{12}>0
$$

Let $c_{12}=\frac{1}{10}$ and $\rho=1$ (the material with elastic constants (6.11) and $c_{12}=0$ is isotropic). Computation of "forbidden" speed by (6.4) yields

$$
c=\sqrt{\frac{12 \sqrt{22}-33}{50}} \approx 0.6824 .
$$

It remains to check whether condition (2.5) is satisfied. But, for waves propagating in the isotropic plane of arbitrary transversely isotropic material,

$$
c_{3}^{\lim }=c_{3},
$$

where $c_{3}$ denotes the minimal speed of all bulk waves propagating in the isotropic plane. For the case considered, computation of $c_{3}$ gives

$$
c_{3}=\sqrt{\frac{c_{55}}{\rho}}=\frac{\sqrt{2}}{2} \approx 0.7071 .
$$

Comparison of the right-hand sides of (6.13) and (6.15) shows that condition (2.5) is satisfied.

c) For the transversely anisotropic material considered in the preceding remark, matrix $\mathbf{R}_{6}$ can be regarded as one-parametric with respect to the phase speed $c$. Analysis of the spectral properties of the matrix $\mathbf{R}_{6}$ in a small vicinity $V$ of the phase speed (6.13), taking account of the notation (4.1)-(4.3), reveals that at $c \rightarrow 0.6824$,

$$
\begin{aligned}
& \gamma_{1} \rightarrow \gamma_{3}, \quad \gamma_{2} \rightarrow \gamma_{4}, \\
& \left(\mathbf{m}_{1}, \mathbf{m}_{1}^{\prime}\right) \rightarrow\left(\mathbf{m}_{3}, \mathbf{m}_{3}^{\prime}\right), \quad\left(\mathbf{m}_{2}, \mathbf{m}_{2}^{\prime}\right) \rightarrow\left(\mathbf{m}_{4}, \mathbf{m}_{4}^{\prime}\right) .
\end{aligned}
$$

It should also be noted that everywhere in $V$, matrix $\mathbf{R}_{6}$ has the Jordan normal form $\mathbf{J}_{6}^{(\mathrm{I})}$, except for the limiting value $c=0.6824$, where the Jordan normal form $\mathbf{J}_{6}^{(\mathrm{II})}$ arises.

At the same time, boundary conditions (5.2), taking account of (6.16), lead to the following relations (at $c \rightarrow 0.6824$ ) between the coefficients $C_{k}$ :

$$
C_{1} \rightarrow-C_{3}, \quad C_{2} \rightarrow-C_{4}, \quad C_{5}=C_{6}=0 .
$$

Combining (6.16), (6.17) with representation (1.2) (which is valid everywhere in $V$, except for the limiting value) yields:

$$
\mathbf{u}(\mathbf{x}) \rightarrow 0
$$

uniformly with respect to $\mathbf{x}$ at $c \rightarrow 0.6824$. In turn, expression (6.18) ensures that both strain and kinetic specific energy functions along with the energy fluxes tend to zero at $c \rightarrow 0.6824$. 
Acknowledgment. The author thanks the INTAS (Grant 96-2306) for financial support.

\section{REFERENCES}

[1] H. Lamb, On waves in an elastic plate, Proc. Roy. Soc. A93, 114-128 (1917)

[2] J. W. Strutt (Lord Rayleigh), On wave propagating along the plane surface of an elastic solid, Proc. London Math. Soc. 17, 4-11 (1885)

[3] G. W. Farnell, Properties of elastic surface waves, Phys. Acoust. 6, 109-166 (1970)

[4] D. M. Barnett and J. Lothe, Synthesis of the sextic and the integral formalism for dislocations, Greens functions, and surface waves in anisotropic elastic solids, Phys. Norv. 7, 13-19 (1973)

[5] P. Chadwick and G. D. Smith, Foundations of the theory of surface waves in anisotropic elastic materials, In: Advances in Applied Mechanics 17, Academic Press, New York, 1977, pp. 303-376

[6] G. T. Mase and G. C. Johnson, An acoustic theory for surface waves in anisotropic media, J. Appl. Mech. 54, 127-135 (1987)

[7] S. A. Gundersen, D. M. Barnett, and J. Lothe, Rayleigh wave existence theory: A supplementary remark, Wave Motion 9, 319-321 (1987)

[8] T. C. T. Ting and D. M. Barnett, Classifications of surface waves in anisotropic elastic materials, Wave Motion 26, 207-218 (1997)

[9] R. Stoneley, Elastic waves at the surface of separation of two solids, Proc. Roy. Soc. London A106, 416-428 (1924)

[10] E. Dieulesaint and D. Royer, Elastic Waves in Solids, John Wiley and Sons, New York, 1980

[11] P. Chadwick and P. Borejko, Existence and uniqueness of Stoneley waves, Geophys. J. Internat. 118, 279-284 (1994)

[12] P. Chadwick, Interfacial and surface waves in pre-strained isotropic elastic media, Z. Angew. Math. Phys. 46, 51-71 (1995)

[13] W. Lin and L. M. Keer, A study of Lamb waves in anisotropic plates, J. Acoust. Soc. Amer. 92, 888-894 (1992)

[14] N. Guo and P. Cawley, Lamb wave propagation in composite laminates and its relationship with acousto-ultrasonics, NDT \& E Internat. 26, 75-84 (1993)

[15] D. E. Chimenti, Lamb waves in microstructured plates, Ultrasonics 32, 255-260 (1994)

[16] O. Poncelet and M. Deschamps, Lamb waves generated by inhomogeneous plane waves, J. Acoust. Soc. Amer. 102, 292-300 (1997)

[17] A. N. Stroh, Steady state problems in anisotropic elasticity, J. Math. Phys. 41, 77-103 (1962)

[18] P. Hartman, Ordinary Differential Equations, John Wiley and Sons, New York, 1964

[19] P. Chadwick, Some remarks on the existence of one-component surface waves in elastic materials with symmetry, Physica Scripta 44, 94-97 (1992) 